\title{
Testosterone Replacement Therapy (TRT) is Associated with Delayed Progression of Chronic Kidney Disease: A Retrospective Analysis of Testosterone Normalization in US Veterans
}

Rishi Sharma, MD, MHSA, Olurinde Oni, MBBS, MPH, Peter Wiegmann, BA, Mukut Sharma*, PhD, Mariana Garcia-Touza, MD, Archana Goel, MD, Virginia Savin, MD, Rajat Barua, MD, PhD, Ram Sharma, MHSA, PhD, and Thomas Wiegmann, MD

Kansas City VA Medical Center, Kansas City, MO, USA

\begin{abstract}
Background: Testosterone replacement therapy (TRT) is used to treat patients with ESRD, but its effects at earlier stages of CKD are unknown.

Study design: Population based retrospective cohort observational study.

Setting and participants: Eligible subjects from Veterans Administration Informatics and Computing Infrastructure (VINCI) were included in the study and divided into two groups namely, (1) Treated to normalize Total Testosterone (TT) and, (2) Untreated.

Predictor: Increase in serum creatinine $(\mathrm{mg} / \mathrm{dl})$ over time was taken as a measure of progression of CKD during follow up (FU) years. Data were analyzed using SPSS and SAS. Individual inversed weight probability matching was used to adjust groups. Results were compared using Kaplan Meier survival curves, hazard ratios (HR) and t-test.

Results: Group 1 normalized TT following TRT ( $\mathrm{N}=38706$, creatinine $1.06 \pm 0.001$, FU 6.1 years) and those in Group 2 maintained low T levels ( $N=9755$, creatinine $1.11 \pm 0.004$, FU 5.1 years). Group 1 showed significant increase in days to reach creatinine to $>1.5$, $(\mathrm{p}<0.0001)$ and $>3.0 \mathrm{mg} / \mathrm{dl}(\mathrm{p}<0.0247)$ indicating delayed progression of CKD. ESRD (creatinine $>6.0 \mathrm{mg} / \mathrm{dl}$ ) was delayed by 284 days (HR 0.734, $p<0.05 \mathrm{vs}$. Group 2). TRT was associated with increase in life span by 328 days (HR $0.753, \mathrm{p}<0.0005$ vs. group 2 ) indicating that TRT delayed death.
\end{abstract}

Limitations: Observational studies may induce unmeasured confounding or hidden bias. There was no randomization due to unavailability of complete clinical data indicating TRT. Thus, the possibility of selection bias cannot be ruled out.

Conclusion: TRT is not associated with significant adverse outcomes in early stages of CKD but rather, with a significant decrease and delay in all-cause mortality, and with delay in progression to ESRD.

Keywords

Testosterone replacement therapy, Chronic kidney disease, Hemodialysis, All-cause mortality

\section{Introduction}

The aging male population suffers from late onset hypogonadism (LOH) with its characteristics including diminished virility, increased fatigue, muscle wasting and decreased bone mass [1-3]. Fatigue, muscle wasting, and diminished libido are also common in advanced chronic kidney disease (CKD), with and without low testosterone [3-5]. Testosterone deficiency is a common feature of failing kidneys and testosterone production is suppressed by multiple causes linked to loss of kidney function [4]. Hypogonadism is the most common gonadal alteration in men
*Corresponding author: Dr. Mukut Sharma, Kansas City VA Medical Center, 4801 E Linwood Blvd, Kansas City, MO 64128, USA, Tel: 816-861-4700, ext 58222

Accepted: January 09, 2020

Published online: January 11, 2020

Citation: Sharma R, Oni O, Wiegmann P, et al. (2020) Testosterone Replacement Therapy (TRT) is Associated with Delayed Progression of Chronic Kidney Disease: A Retrospective Analysis of Testosterone Normalization in US Veterans. Ann Nephrol 5(1):51-59 
Citation: Sharma R, Oni O, Wiegmann P, et al. (2020) Testosterone Replacement Therapy (TRT) is Associated with Delayed Progression of Chronic Kidney Disease: A Retrospective Analysis of Testosterone Normalization in US Veterans. Ann Nephrol 5(1):51-59

with CKD and with end-stage renal disease (ESRD) [6]. Up to two thirds of ESRD patients have low testosterone levels and TRT has been shown to improve sexual function, muscle mass and bone mineral density [7].

The Endocrine Clinical Practice Guideline published in June 2010 recommended assessment of testosterone levels of patients with end-stage renal disease. Therapy with testosterone replacement is recommended in this population but there is no apparent generally accepted therapeutic practice for use of TRT to treat those with CKD.

Hypogonadism is defined by low serum testosterone concentration along with inappropriately normal or low luteinizing hormone (LH) level (3) with combined effects of inflammatory, metabolic, and hormonal mediators at various levels of the hypothalamic-pituitary-testicular axis [3,8,9]. Multiple biological pathways are affected by, and may contribute to hypogonadism by affecting several mechanisms including inhibition of luteinizing hormone signaling, reduced prolactin clearance by kidneys [10] and inhibition of regulatory synthesis of testosterone and other sex hormones. Chronic use of Angiotensin converting enzyme inhibitors (ACEI), angiotensin receptor blockers (ARB), spironolactone, ketoconazole and glucocorticoids [4] also contributes to hypogonadism.

To understand if low levels of total testosterone (TT) are associated with the progression of CKD and whether normalization of TT levels by TRT can slow the progression of CKD, we examined the relationship between renal function, total testosterone levels (TT) and TRT in a large cohort of veterans. Results of this observational study suggest that TRT delays progression of CKD, prolongs the time to ESRD and mortality.

\section{Methods}

\section{Data Source}

Data were obtained from the Veterans Administration Informatics and Computing Infrastructure (VINCI). The VINCI database comprises clinical data obtained from over 1,400 Veterans Health Administration (VHA) establishments across the United States. Clinical data from these establishments are archived in the Corporate Data Warehouse (CDW) and provided for VA researchers upon request. The Institutional Review Board of Kansas City Veterans Affairs Medical Center, MO, USA approved the study.

\section{Ascertainment of Total Testosterone (TT) level}

Testosterone levels were considered low at reported TT values less than the lower limit of normal laboratory reference range of the test. This method was preferred over the use of discrete cut-off values because we found that reference ranges varied with the assays used during the study period at different facilities across the VA $[11,12]$.

\section{Study Population}

The initial population of 83,000 subjects with low TT-levels has been described previously [13]. We included subjects with $\mathrm{TT}$ level lower than the respective normal laboratory reference range (NLRR) in the first laboratory assay for TT.
Table 1: Study Population and the number of subjects in groups.

\begin{tabular}{|l|l|l|}
\hline Groups & \multicolumn{2}{|l|}{ Number and (\%) } \\
\hline Initial Cohort with low Total Testosterone & 83000 & \\
\hline With Baseline Creatinine data, N (\%) & $48461(100)$ & \\
\hline Treated N (\%) & $38706(79.8)$ & Group 1 \\
\hline Untreated N (\%) & $9755(20.2)$ & Group 2 \\
\hline
\end{tabular}

Note: Data from subjects showing low total testosterone (TT) were first selected. Those with initial creatinine data were selected for analyses. From this population (100\%), those who received TRT $(79.2 \%)$ were included in Group 1 and untreated subjects were included in Group 2. Treated and untreated groups were expressed as absolute numbers and as percentages of those enrolled into this study.

Abbreviations: N: Number; \%: Percent.

We excluded women and subjects who had received TRT before the first laboratory finding of low TT. The present study comprises a subset of 48,461 subjects with data on baseline serum creatinine levels to enable us to determine their renal function. 38,706 subjects received some form of TRT (injection, gel or patch) and subsequently had normalized TT-levels. 9,755 subjects received no treatment and retained low TT-levels. The distribution of subjects into two groups is illustrated in Table 1.

\section{Outcome variables}

Serum creatinine data were collected in three-month bracket intervals. The date of the laboratory test was used to calculate the elapsed time from the date of first treatment. This was taken as the baseline date. In the untreated group the date of low TT-level was used as the baseline date. In the case of multiple lab values per 3-month time interval we calculated the average and assigned it to the last date point in the period. Some 3-month intervals contained no creatinine data. Estimated glomerular filtration rate (eGFR) was calculated using the Chronic Kidney Disease Epidemiology Collaboration (CKD-EPI, GFR-EPI) equation as described by Coresh, et al. [14]. Where race data were missing, the formula for non-black was used. This approach was used for the initial staging of CKD. GFR-EPI in this text refers to eGFR values calculated using this equation.

We determined the time to increase in creatinine to more than $1.5 \mathrm{mg} / \mathrm{dl}$, and subsequent doubling to above $3.0 \mathrm{mg} / \mathrm{dl}$, and doubling above $6.0 \mathrm{mg} / \mathrm{dl}$, respectively. A creatinine value of $>6 \mathrm{mg} / \mathrm{dl}$ was taken to represent ESRD. Corresponding hemodialysis data in the VINCI database were incomplete and therefore, were not used. Survival was determined by the difference in days from baseline-date to death-date. Additional data included body weight, height and $\mathrm{BMI}$, blood pressure as well as hematocrit, hemoglobin A1c (HbA1c) and LDL levels. The ICD-9-CM diagnosis codes, laboratory and pharmacy records were used to capture these coexisting conditions.

\section{Statistical Analysis}

Data were analyzed using SAS Enterprise Guide 7.1 as 
Citation: Sharma R, Oni O, Wiegmann P, et al. (2020) Testosterone Replacement Therapy (TRT) is Associated with Delayed Progression of Chronic Kidney Disease: A Retrospective Analysis of Testosterone Normalization in US Veterans. Ann Nephrol 5(1):51-59

provided by $\mathrm{VINCl}$. We used propensity score-weighted stabilized inverse probability of treatment weights (IPTW) for multivariate analyses. This method ensures that the groups being compared are well-matched. Use of IPTW also enables inclusion of the highest number of subjects in the study after matching. It enhances a robust analysis and achieves a balance between each pair of sub-groups studied.

Stabilized IPTW (SIPTW) involves weighting individuals by the inverse probability of their treatment status. SIPTW is a preferred approach over the regular IPTW or propensity score matching (PSM) because SIPTW enhances inclusion of data on the largest number of subjects for analysis, whereas PSM may result in a decreased sample size. SIPTW also significantly corrects for the instability in estimated treatment weights that potentially results from the use of regular IPTW for individuals with very low probability of treatment $[15,16]$. The COX proportional hazard model was used to correct for potential systemic difference in the evaluation of treatment effects.

The model was adjusted for conditions affecting progression of kidney disease such as age, BMI, presence of hypertension, proteinuria, race and treatment with ACE or ARB (Table 2). We compared treated vs. untreated population characteristics using $t$-test and chi-square test $\left(\chi^{2}\right.$-distribution). Folded F-test was used to check equality of variance and Satterthwaite $p$-values were used for unequal variance. Analysis of categorical values was performed using the standard chi-square test and p-value. Kaplan-Meier estimates and hazard ratios were calculated to evaluate the risk of progression and death.

\section{Results}

LOH was confirmed by repeated laboratory assays of low total $T T$ levels and propensity-matched comparison of renal function was performed between subjects who received TRT and normalized TT (Group 1) and those who received no treatment and retained low TT levels (Group 2) as shown in Table 1.

Table 2: Frequency distribution of factors that likely influence the progression of renal disease.

\begin{tabular}{|c|c|c|c|c|c|c|c|c|}
\hline Treatment Group & & No & Yes & & No & Yes & & chi-square \\
\hline Variable present & & No & No & Total & Yes & Yes & Total & $p$-value \\
\hline \multirow[t]{2}{*}{ Age $\geq 50$} & $N$ & 1866 & 7337 & 9204 & 7889 & 31369 & 39258 & 0.6927 \\
\hline & $\%$ group & 19.13 & 18.96 & & 80.87 & 81.04 & & \\
\hline \multirow[t]{2}{*}{$\mathrm{BMI} \geq 30$} & $\mathrm{~N}$ & 350 & 13487 & 16838 & 6405 & 25219 & 31624 & 0.3514 \\
\hline & $\%$ group & 34.34 & 34.85 & & 65.66 & 65.15 & & \\
\hline \multirow[t]{2}{*}{ Hypertension } & $\mathrm{N}$ & 8880 & 35261 & 44140 & 876 & 3446 & 4321 & 0.8199 \\
\hline & $\%$ group & 91.02 & 91.1 & & 8.98 & 8.9 & & \\
\hline \multirow[t]{2}{*}{ Diabetes Mellitus 2} & $\mathrm{~N}$ & 6687 & 26557 & 33244 & 3068 & 12150 & 15217 & 0.9119 \\
\hline & $\%$ group & 68.55 & 68.61 & & 31.45 & 31.39 & & \\
\hline \multirow[t]{2}{*}{ Uprot $\geq 30 \mathrm{mg}$} & $\mathrm{N}$ & 8200 & 32529 & 40730 & 1555 & 6177 & 7732 & 0.9617 \\
\hline & $\%$ group & 84.06 & 84.04 & & 15.94 & 15.96 & & \\
\hline \multirow[t]{2}{*}{ Self ID as Black } & $N$ & 9385 & 37228 & 46613 & 370 & 1478 & 1848 & 0.8959 \\
\hline & $\%$ group & 96.21 & 96.18 & & 3.79 & 3.82 & & \\
\hline \multirow[t]{2}{*}{ ACE or ARB } & $\mathrm{N}$ & 5669.83 & 22481.2 & 28151.1 & 4085.27 & 16224.9 & 20310.1 & 0.9432 \\
\hline & $\%$ group & 58.12 & 58.08 & & 41.88 & 41.92 & 41.91 & \\
\hline
\end{tabular}

Note: These factors (left column) were used in the propensity analysis (inversed individual weight) matching. Results were grouped by the presence/absence of each factor and the treatment group.

Abbreviations: BMI: Body mass index; Uprot: Urinary protein; ID: Identification (race/ethnic); ACE: Angiotensin-converting-enzyme; ARB: Angiotensin-receptor blocker.

Table 3: eGFR calculated using the EPI formula compared with eGFR reported by VA laboratories and archived (VINCI Database).

\begin{tabular}{|l|l|l|l|l|l|l|l|}
\hline & \multicolumn{3}{l}{ No Treatment } & \multicolumn{3}{l|}{} & \\
\hline Variable & Mean & SEM & N & Mean & SEM & N & $t$-test \\
\hline & & & & & & & $p$-value \\
\hline eGFR EPI Start & 82.71 & 0.24 & 9689 & 82.69 & 0.11 & 38408 & $<0.94$ \\
\hline GFR VA-Lab Start & 82.71 & 0.3 & 5666 & 81.41 & 0.14 & 21190 & $<0.0001$ \\
\hline
\end{tabular}

Note: Different N for VA-Lab when calculated GFR was not provided p-values compared the eGFR (EPI) and GFR (VA lab) at the beginning of the study between the testosterone replacement therapy group versus the untreated group.

Abbreviations: eGFR: Estimated glomerular filtration rate; EPI: Short form for Chronic Kidney Disease Epidemiology Collaboration (CKD-EPI) equation; GFR: Glomerular filtration rate. 
Citation: Sharma R, Oni O, Wiegmann P, et al. (2020) Testosterone Replacement Therapy (TRT) is Associated with Delayed Progression of Chronic Kidney Disease: A Retrospective Analysis of Testosterone Normalization in US Veterans. Ann Nephrol 5(1):51-59

Results show that TRT is associated with significant delay in the progression of CKD and significant reduction in all-cause mortality. We included variables that affect the progression of kidney disease and renal function, namely, age, BMI, diagnosis of hypertension and/or diabetes, treatment with ACE or $A R B$, initial CKD stage and presence of significant proteinuria (>= $30 \mathrm{mg} /$ day). Inclusion of race was limited by the fact that racial characteristics are self-reported within the VA system and data are frequently incomplete. As shown in Table 2, use of IPTW resulted in a balanced comparison between populations where the differences between treated and untreated groups were not significant for every confounding variable.

Laboratories in the VA system generally calculate GFR (GFR-VA) using the Modification of Diet in Renal Disease (MDRD) Study equation. Results of GFR-VA at baseline were similar to our calculated GFR-EPI in untreated subjects $(82.7$ $+0.3 \mathrm{mg} / \mathrm{dl})$ and treated subjects $(81.4 \pm 0.1$ ) (Table 3). We did not use GFR-VA because it was reported in the VINCI database as a value separate from creatinine only for small subsets of 5,666 untreated and 21,190 treated subjects.

The baseline distribution of study subjects by CKD stages 1-5 is given in Table 4. Our combined study population comprised a large proportion of subjects with kidney disease. Only $35.3 \%$ of study subjects were at Stage 1 compared to $64.30 \%$ in the general population as previously described [14]. Diminished function was more common in our study with Stage 2 (50.6\% vs. 31.2\%) compared to that in general US population. Likewise, for Stage 3 the proportion was significantly higher in the present study $(13.3 \%$ vs. $4.3 \%)$ and again with Stage $4(0.79 \%$ vs. $0.2 \%)$ [14]. Number of subjects in the study population at Stage 5 was approximately similar at $0.17 \%$ in this analysis versus $0.2 \%$ in the general population. Following IPTW, no significant differences were observed among the treated and untreated groups and various CKD stages $(p=0.9846)$. A post hoc

Table 4: Different stages of CKD per eGFR calculated by EPI formula at the start of study.

\begin{tabular}{|c|c|c|c|c|c|}
\hline \multirow{3}{*}{\begin{tabular}{|l} 
CKD Stage \\
1 \\
\end{tabular}} & \multirow[b]{3}{*}{$\mathrm{N}$} & \multirow{3}{*}{\begin{tabular}{|l|} 
No \\
Treatment \\
3466 \\
\end{tabular}} & Yes & \multicolumn{2}{|c|}{ Combined } \\
\hline & & & \multicolumn{2}{|c|}{ Treatment } & \multirow[t]{2}{*}{ p-value } \\
\hline & & & 13624 & 17090 & \\
\hline & $\%$ Group & 35.53 & 35.2 & 35.27 & \\
\hline \multirow[t]{2}{*}{2} & $N$ & 4914 & 19602 & 24517 & \\
\hline & $\%$ Group & 50.38 & 50.64 & 50.59 & \\
\hline \multirow[t]{2}{*}{3} & $N$ & 1291 & 5145 & 6435 & \\
\hline & $\%$ Group & 13.23 & 13.29 & 13.28 & \\
\hline \multirow[t]{2}{*}{4} & $\mathrm{~N}$ & 67 & 268 & 335 & \\
\hline & $\%$ Group & 0.69 & 0.69 & 0.69 & \\
\hline \multirow[t]{2}{*}{5} & $N$ & 17 & 67 & 84 & \\
\hline & $\%$ Group & 0.17 & 0.17 & 0.17 & \\
\hline \multirow[t]{2}{*}{ Total } & $N$ & 9755 & 38706 & 48461 & 0.9846 \\
\hline & $\%$ Group & 20.13 & 79.87 & 100 & \\
\hline
\end{tabular}

Note: ANOVA p-value overall, 0.9846 (no post-hoc comparisons per separate stages). Differences between treatment groups for stages were compared using ANOVA. No post-hoc comparisons per separate stages.

Abbreviations: CKD: Chronic kidney disease; N: Number.

Table 5: Comparison of clinical parameters pre- and post-trt.

\begin{tabular}{|c|c|c|c|c|c|c|c|}
\hline \multirow[t]{3}{*}{ Variable } & \multirow{3}{*}{$\begin{array}{l}\text { No } \\
\text { Treatment } \\
\text { Mean }\end{array}$} & \multirow[t]{3}{*}{ SEM } & \multirow{3}{*}{$\mathbf{N}$} & \multirow{3}{*}{$\begin{array}{l}\text { Yes } \\
\text { Treatment } \\
\text { Mean }\end{array}$} & \multirow[t]{3}{*}{ SEM } & \multirow{3}{*}{$\mathbf{N}$} & \\
\hline & & & & & & & $t$-test \\
\hline & & & & & & & $p$-value \\
\hline LDL mg/dl & -9.96 & 0.38 & 8120 & -12.99 & 0.2 & 32111 & $<0.0001$ \\
\hline A1c \% & -0.03 & 0.01 & 7522 & 0.01 & 0.01 & 31853 & $<0.0056$ \\
\hline Alb/Creat & 28.57 & 7.07 & 2804 & 26.22 & 2.58 & 10309 & $<0.0001$ \\
\hline hct \% & 1.03 & 0.05 & 9484 & 0.4 & 0.03 & 37841 & $<0.0001$ \\
\hline BP_Syst & -3.78 & 0.23 & 9746 & -2.6 & 0.11 & 38705 & $<0.04$ \\
\hline BP_diast & -2.9 & 0.14 & 9745 & -2.43 & 0.07 & 38705 & $<0.0001$ \\
\hline
\end{tabular}

Note: Mean differences were compared between treated and untreated groups using t-test.

Abbreviations: LDL: Low density lipoproteins; A1C: Hemoglobin A1C; Alb/Creat: Urinary albumin creatinine ratio; hct: Hematocrit; BP syst: Systolic blood pressure; BP diast: Diastolic blood pressure. 
Citation: Sharma R, Oni O, Wiegmann P, et al. (2020) Testosterone Replacement Therapy (TRT) is Associated with Delayed Progression of Chronic Kidney Disease: A Retrospective Analysis of Testosterone Normalization in US Veterans. Ann Nephrol 5(1):51-59

Table 6: Days to event and numbers at risk.

\begin{tabular}{|l|l|l|l|l|l|l|l|}
\hline Event & Group & N & Days & SE & p-value & Hazard Ratio & p-value \\
\hline Death & No Rx & 816 & 1345.2 & 34.9 & & & $<0.0005$ \\
\hline & TRT Rx & 1509 & 1673.4 & 26.1 & $<0.0001$ & 0.753 & \\
\hline Creat $>=1.5$ & No Rx & 1815 & 794.6 & 19.7 & & & $<0.0005$ \\
\hline Creat $>=3.0$ & TRT Rx & 8036 & 885.6 & 9.7 & $<0.0001$ & 0.905 & \\
\hline & No Rx & 335 & 1109 & 54.7 & & & $<0.05$ \\
\hline Creat $>=6.0$ & TRT Rx & 1146 & 1279.5 & 31.5 & $<0.02$ & 0.857 & \\
\hline & No Rx & 66 & 1029.4 & 102 & & & $<0.05$ \\
\hline
\end{tabular}

Note: Summary of mean number of days $( \pm$ SEM) to death or to creatinine to $>=1.5,3.0$ and 6.0 are shown with $p$-value. Abbreviations: N: Number; SE: Standard error of the mean; Creat: Creatinine.

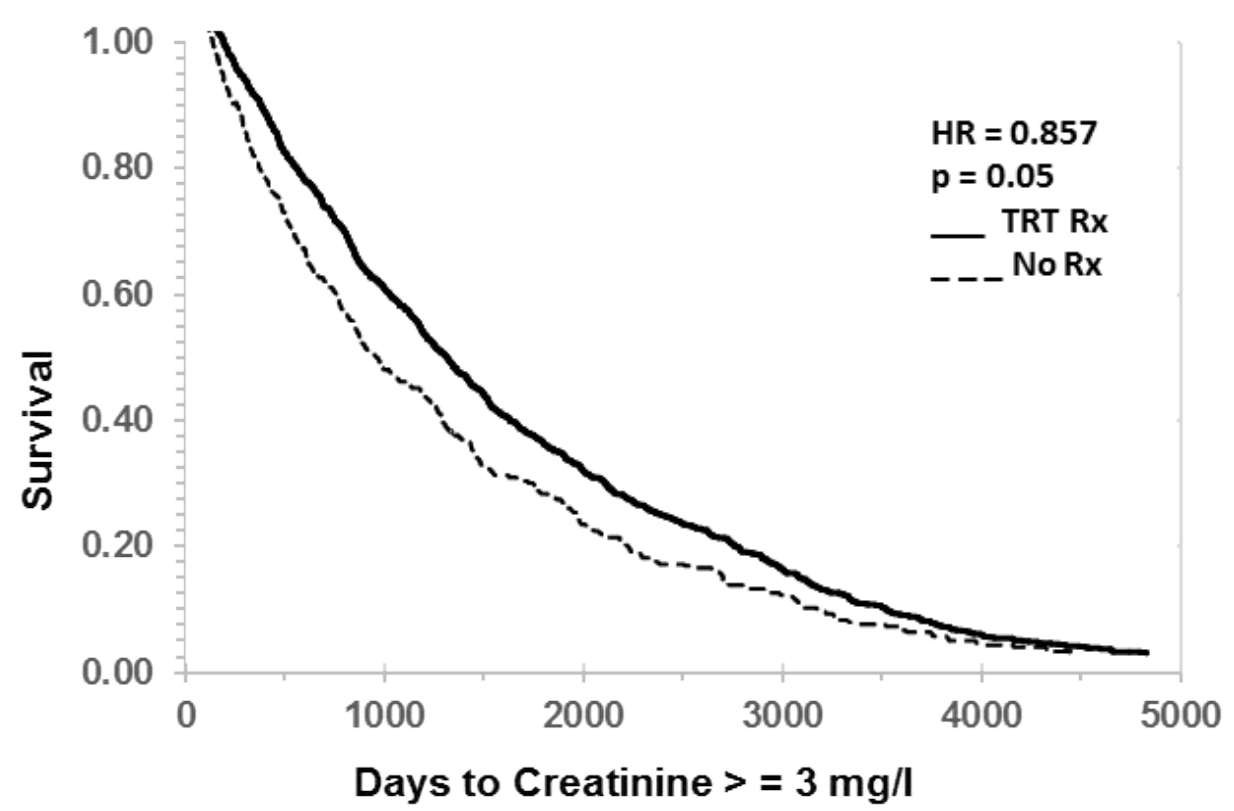

Figure 1: Effect of TRT on creatinine increase from baseline to $>=3 \mathrm{mg} / \mathrm{d}$.

TRT delayed the time (days) to increase in creatinine from baseline to $>=1.5 \mathrm{mg} / \mathrm{dl}$. Data from TRT treated group and untreated group were used to obtain Kaplan Meier plots to compare the time (days) taken from baseline to $>=3 \mathrm{mg} / \mathrm{dl}$ over time. Results showed that TRT was associated with a significant delay in creatinine increase $(\mathrm{HR}=0.857 ; p<0.05)$.

analysis by separate CKD stage was therefore not done.

The effect of TRT on blood pressure (BP) and selected laboratory values is shown in Table 5, which describes the differences between the start and finish for the TRT and the control groups. Over the course of the study, it was found that BP improved in both groups. Likewise, LDL decreased; diabetes control remained unaffected and; hematocrit was lower in the TRT group. A small increase in urinary albumin creatinine ratio (UACR) was also seen in both groups.

Table 6 provides a summary of the key findings on the numbers of subjects and days to reach creatinine $>=1.5,3.0$ or 6.0 as well as the numbers and days to all-cause mortality.

The survival function of untreated and treated study populations is presented as Kaplan Meier plots (Figure 1, Figure 2 and Figure 3 ) to illustrate the effect of TRT on CKD progression. A standard measure of disease progression such as in- crease in creatinine of $30 \%$ over baseline was not significantly different (HR 0.959, N.S., data not shown). Also, the doubling of creatinine from baseline was also not significantly different between the two groups (HR 1.003, N.S., data not shown).

In contrast, increase in creatinine from baseline to greater than $1.5 \mathrm{mg} / \mathrm{dl}$ was significantly delayed by TRT (Table 6, HR $0.905, p<0.0005)$. Likewise, doubling of creatinine to greater than $3 \mathrm{mg} / \mathrm{dl}$ was also delayed in those who received TRT (Figure 1 and Table 6, HR 0.857, p<0.05). Significant delay in progression to ESRD following TRT was indicated by creatinine doubling again to greater than $6 \mathrm{mg} / \mathrm{dl}$ as shown in Figure 2 (HR 0.734, p < 0.05).

The effect of TRT on all-cause mortality is shown as Kaplan Meier plot in Figure 3. The delaying effect of TRT was highly significant ( $p<0.0001)$. Death occurred in 1509 cases in the TRT group and in 816 cases in non-TRT group. TRT delayed 
Citation: Sharma R, Oni O, Wiegmann P, et al. (2020) Testosterone Replacement Therapy (TRT) is Associated with Delayed Progression of Chronic Kidney Disease: A Retrospective Analysis of Testosterone Normalization in US Veterans. Ann Nephrol 5(1):51-59

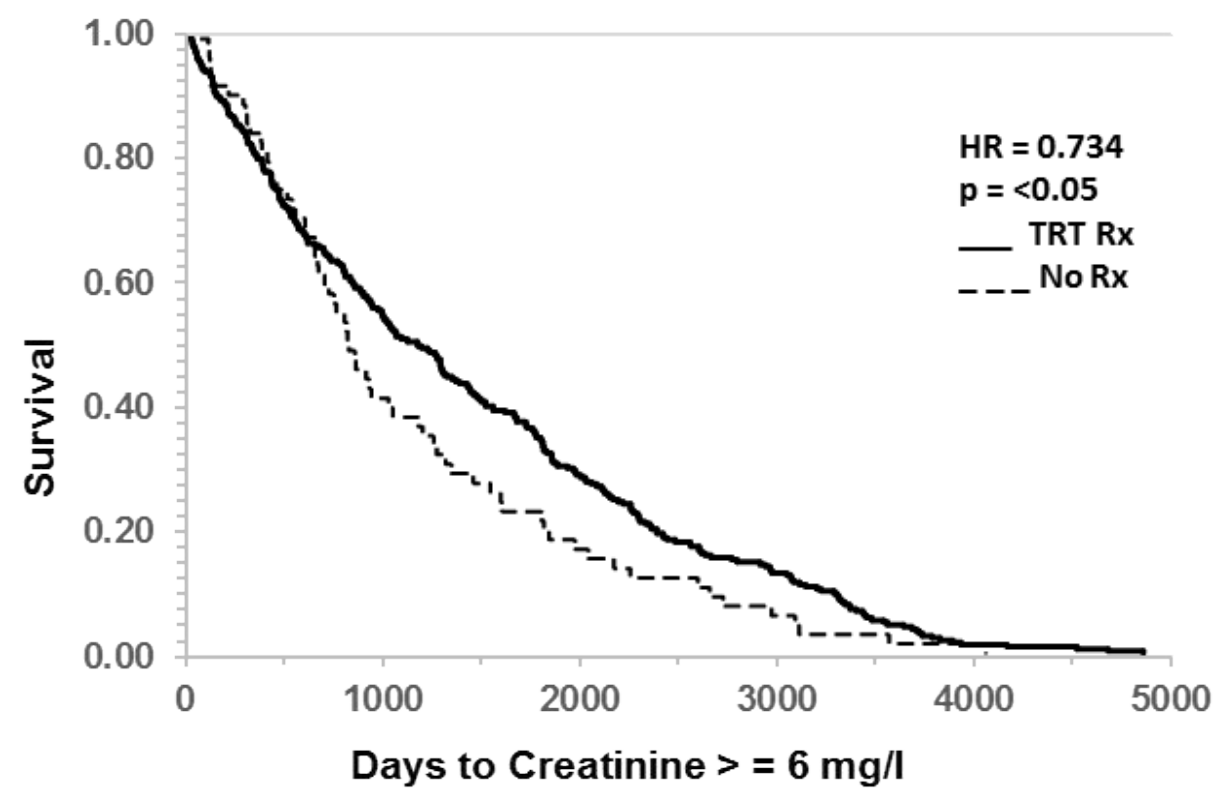

Figure 2: Effect of TRT on increase in creatinine from baseline to $>=6 \mathrm{mg} / \mathrm{d}$.

TRT delayed the increase in creatinine from baseline to $>=6 \mathrm{mg} / \mathrm{dl}$. Data from TRT treated group and untreated groups were used to obtain Kaplan-Meier plots. Results showed that the TRT group showed a significant delay in increased creatinine from baseline to above $6.0 \mathrm{mg} / \mathrm{dl}$ over time $(\mathrm{HR}=0.734 ; p<0.05)$.

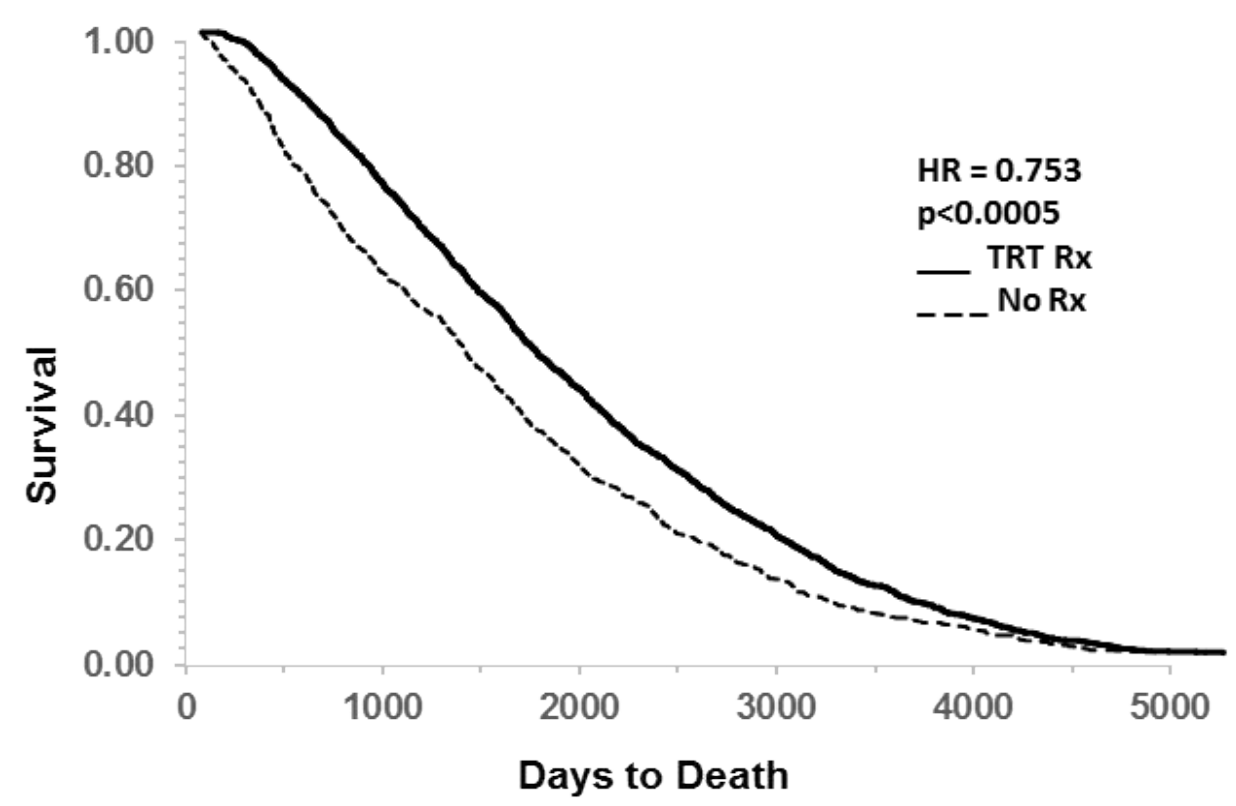

Figure 3: Effect of TRT on all-cause mortality over time.

TRT resulted in delayed all-cause mortality. Kaplan Meier curves were obtained from data in treated and untreated groups. A significant delay in all-cause mortality was observed in the treated group compared to the untreated group $(\mathrm{HR}=0.753 ; p<0.0005)$.

death by an average of 693 days and the HR was significantly reduced to 0.753 ( $p<0.0005$ ). Taking into consideration the difference in group sizes, these numbers represent a $75 \%$ reduction in all-cause mortality.

\section{Discussion}

We examined the effect of TRT on renal function over time in this retrospective analysis of the data on 48,461 vet- erans with a laboratory diagnosis of $\mathrm{LOH}$ and available renal function data. Our results document that TRT was associated with a significant delay in the progression of kidney disease and reduced all-cause mortality. In addition, using the ESRD surrogate of transition above $6 \mathrm{mg} / \mathrm{dl}$ creatinine we found that TRT significantly delayed ESRD by 1,509 days. A similar effect was present at earlier stages of disease progression as exemplified by increases in creatinine above threshold levels of $1.5 \mathrm{mg} / \mathrm{dl}$ and subsequent doubling to 3.0 and $6.0 \mathrm{mg} / \mathrm{dl}$ 
Citation: Sharma R, Oni O, Wiegmann P, et al. (2020) Testosterone Replacement Therapy (TRT) is Associated with Delayed Progression of Chronic Kidney Disease: A Retrospective Analysis of Testosterone Normalization in US Veterans. Ann Nephrol 5(1):51-59

(Table 6). A common measure of injury such as $30 \%$ increase in creatinine from baseline was not significantly different after TRT. We were unable to determine whether an increase of $30 \%$ represented an acute event with subsequent recovery, or simply a stage of progression of CKD. Likewise, doubling of creatinine from baseline did not show a significant relation with TRT. These results were not included for presentation.

All-cause mortality was found to be significantly reduced in the TRT-treated group. This echoes the finding in our initial study population of 83,010 subjects [13]. In that study, confounding factors were essentially cardiovascular and included consideration for the use of beta-blockers statins, and anti-hypertensive agents as well as prior vascular diseases. In the present study, we focused on renal confounding factors instead and obtained similar results.

There are various pathways that connect testosterone to vascular functions and inflammatory processes that may affect progression of kidney disease. Androgens have been shown to increase proximal tubular sodium and water reabsorption which, in turn, activates the renin-angiotensin and endothelin systems [17]. Some animal models have shown pro-inflammatory effects for testosterone during kidney injury. This then would perhaps promote vascular and renal disease with TRT. On the other hand, testosterone concentrations are inversely related to the concentration of C-reactive protein (CRP), interleukin-6 (IL-6), and fibrinogen. Thus, inflammatory markers are higher in hypogonadic men with CKD [18] and TRT may be beneficial.

Previously, a smaller clinical study that included thirty-three patients with hypogonadotropic hypogonadism compared to 37 healthy subjects showed correlation between idiopathic hypogonadotropic hypogonadism, glomerular hyperfiltration and development of proteinuria [19]. Changes in glomerular endothelial structure and function due to various systemic or local factors can lead to albuminuria [6]. Cases of CKD with low levels of free testosterone are associated with a higher risk of CV related endpoints. Given the similarity of symptoms between $\mathrm{LOH}$ and advancing CKD, it is important to know the true prevalence of $\mathrm{LOH}$ in the CKD population. It is unclear how TRT would benefit late CKD or ESRD patients with proven low testosterone levels. Excellent publications review the available information on treatment options. However, there appears to be no agreement on the use of TRT at early stages of CKD and some investigators advise against it $[20,21]$.

Data on the safety of TRT are equivocal and this lack of clarity may explain the physician's general reluctance to consider TRT, particularly for CKD patients. Recent studies reported an increased risk of myocardial ischemia (MI), stroke, and other events as well as increased mortality associated with TRT [22-25]. Vigen's retrospective analysis showed an association between TRT and all-cause mortality, stroke, and MI among 8,709 VA patients with and without CAD [22]. However, the study only included hypogonadal men who had undergone coronary angiography which represents exclusive focus on a high-risk population. In this study, since compliance to TRT was not assessed and $100 \%$ adherence was assumed, it is possible that compliance bias may have affected the results. Finkle, et al. reported increased rates of non-fatal MI within 90 days of a TRT prescription being filled, as compared to the 12 months prior to TRT [23]. These authors reported results for only a very short duration, worked with a retrospective data set without a control group, compared dissimilar groups and found a relatively small increase in the risk of MI. Numerous problems with methods and conclusions in these studies have been identified and detailed by Morgentaler $[26,27]$. Basaria, et al. found an association between TRT and increased risk of cardiovascular events [24]. The study only included a small number of men with limitations in mobility and high levels of chronic disease. More recently Budoff, et al. reported an increase in uncalcified arterial plaque volume without coincidental increase in cardiovascular events in the NIH Testosterone Trials [28].

In contrast, our group has reported significant cardio-protective effects of TRT, at least in veterans with documented LOH. We found that normalization of TT levels in our initial base population after TRT was associated with reduction in all-cause mortality, MI, and stroke. TRT was also associated with significant reduction in atrial fibrillation. TRT showed a significant association with DVT and pulmonary embolism $[13,29,30]$. Our present study represents a subset of that base population with laboratory data on renal function. Various described cardiovascular benefits seen in the base population can be presumed to be present in our subset as well.

Others have reported similar mortality and cardiovascular findings in smaller studies. Shore, et al. reported reduced mortality with TRT [31]. Baillargeon, et al. found that TRT was not associated with a higher risk of MI compared to no-TRT [32]. A study by Zhao, et al. revealed that low plasma testosterone may be a risk factor for coronary artery disease while Muraleedharan, et al. found that low testosterone levels may predict increased all-cause mortality $[33,34]$.

Another area of vascular risk with potential effect on renal circulation relates to polycythemia with an increased risk of thrombosis as a recognized effect of TRT. A meta-analysis of 19 studies found a significant increase in hematocrit to $>50$ as the most frequent adverse event $[35,36]$. Its mechanism has only recently become clearer with the recognition that TRT may block hepcidin [37]. In extension, anemia during hemodialysis (HD) is, in part, independent of testosterone as it affects both men and women. However, the demand for EPO in HD men is lower than in HD women $[38,39]$. TRT may show a significant benefit in reducing EPO requirements in $\mathrm{LOH}$ patients undergoing HD. Therefore, this relationship merits $A$ further investigation based on patients with more advanced CKD. It is therefore of interest to note that in our population, even with increased prevalence of CKD stage 3 and 4, we did not find significant differences in hematocrit response between groups.

Reduction in visceral obesity is considered an important effect of TRT, because insulin resistance and glucose tolerance are impacted negatively by obesity [40]. Substantial evidence from double blind placebo controlled studies indi- 
Citation: Sharma R, Oni O, Wiegmann P, et al. (2020) Testosterone Replacement Therapy (TRT) is Associated with Delayed Progression of Chronic Kidney Disease: A Retrospective Analysis of Testosterone Normalization in US Veterans. Ann Nephrol 5(1):51-59

cates that TRT improves insulin resistance as measured by the Homeostatic Model of Insulin Resistance (HOMA-IR) and decreases $\mathrm{HbA} 1 \mathrm{c}$ by $0.8 \%$ over a period of 18 months. TRT improved general metabolism measured by $\mathrm{HbA1c}$, total cholesterol, and waist circumference in men with type 2 diabetes. Of interest, results were markedly decreased in men with depression at baseline [41]. There is evidence of decreased LDL-cholesterol and sexual health in hypogonadal men with DM2 [42]. TRT increased insulin sensitivity by $25 \%$, with improved glycemic control, as well as lower LDL cholesterol and reduced visceral adiposity [43]. Overall this associates with increased mortality [33]. In our study, blood pressure and lipid control improved over the course of the study in both TRT-treated and untreated subjects.

Altogether, in our study the use of TRT was found to be associated with a significant and beneficial effect against the progression of CKD and all-cause mortality in aging men with documented LOH. Notably, the effect was present in a population that had a higher proportion of CKD stage 2-4 compared to the general population.

Our study had its limitations, some of them were inherent to it being a retrospective study. Large sample size and application of propensity based statistical methods with consideration of various confounding factors that relate to progression of kidney disease would limit these concerns. Moreover, we did not find the expected polycythemia and cannot make a definitive statement on the effect of TRT on anemia. Likewise, we did not detect a beneficial effect of TRT on diabetes control. Our study does not provide data on the prevalence of LOH with CKD.

We believe that our findings underscore the need to study the prevalence of $\mathrm{LOH}$ in our CKD population, especially in patients with signs and symptom of $\mathrm{LOH}$. In the present study, TRT did not promote progression of kidney disease. Indeed, TRT may provide wide ranging benefits that include EPO-sparing effects, increased muscle and bone mass with reduced fracture rates as well as better glycemic control of a large diabetic population with CKD. So far, it has been difficult to show uniform symptomatic benefits and the testosterone trials have failed to show effect on vitality while improving sexual function and depression [44]. Even so, a further look is indicated and an effective symptomatic treatment of the uremic general malaise would be welcome. Altogether, we believe that patients with CKD stage 4 and 5 should be evaluated for $\mathrm{LOH}$ and treatment should be considered for documented $\mathrm{LOH}$. More work is needed to determine the utility of early interventions with TRT in patients with an earlier stage of CKD.

\section{Acknowledgements}

\section{Disclaimer}

Views expressed in this article are those of the authors and do not necessarily reflect the position or policy of the Department of Veterans Affairs or the United States government.

\section{Support}

We gratefully acknowledge the support of the Veterans Administrations Informatics and Computing Infrastructure (VINCI) in acquiring the data.

\section{Financial Support}

MS received support, in part, through grants from the National Institutes of Health (NIH-NIDDK R01DK107490) and the Veterans Administration (VA BX001037) and through the Midwest Veterans' Biomedical Research Foundation, Kansas City, MO.

\section{Financial Disclosure}

Authors do not have competing financial interests to declare.

\section{References}

1. Bhasin S, Storer T (2009) Anabolic applications of androgens for functional limitations associated with aging and chronic illness. Front Horm Res 37: 163-182.

2. Corona G, Rastrelli G, Maggi M (2013) Diagnosis and treatment of late-onset hypogonadism: Systematic review and meta-analysis of TRT outcomes. Best Pract Res Clin Endocrinol Metab 27: 557-579.

3. Doumouchtsis KK, Perrea DN, Doumouchtsis SK (2009) The impact of sex hormone changes on bone mineral deficit in chronic renal failure. Endocr Res 34: 90-99.

4. Carrero JJ, Stenvinkel P (2012) The vulnerable man: Impact of testosterone deficiency on the uraemic phenotype. Nephrol Dial Transplant 27: 4030-4041.

5. Dousdampanis P, Trigka K, Fourtounas C, et al. (2014) Role of testosterone in the pathogenesis, progression, prognosis and comorbidity of men with chronic kidney disease. Ther Apher Dial 18: 220-230.

6. Bao Y, Johansen KL (2015) Diagnosis and treatment of low testosterone among patients with end-stage renal disease. Semin Dial 28: 259-265.

7. Iglesias P, Carrero JJ, Diez JJ (2012) Gonadal dysfunction in men with chronic kidney disease: Clinical features, prognostic implications and therapeutic options. J Nephrol 25: 31-42.

8. Wang C, Jackson G, Jones TH, et al. (2011) Low testosterone associated with obesity and the metabolic syndrome contributes to sexual dysfunction and cardiovascular disease risk in men with type 2 diabetes. Diabetes Care 34: 1669-1675.

9. Liel $Y(2017)$ Clomiphene citrate in the treatment of idiopathic or functional hypogonadotropic hypogonadism in men: A case series and review of the literature. Endocr Pract 23: 279-287.

10. Cobo G, Hecking M, Port FK, et al. (2016) Sex and gender differences in chronic kidney disease: Progression to end-stage renal disease and haemodialysis. Clin Sci (Lond) 130: 1147-1163.

11. Lazarou S, Reyes-Vallejo L, Morgentaler A (2006) Wide variability in laboratory reference values for serum testosterone. J Sex Med 3: 1085-1089.

12. Rosner W, Auchus RJ, Azziz R, et al. (2007) Position statement: Utility, limitations, and pitfalls in measuring testosterone: An Endocrine Society position statement. J Clin Endocrinol Metab 92: 405-413.

13. Sharma R, Oni OA, Gupta K, et al. (2015) Normalization of testosterone level is associated with reduced incidence of myocardial infarction and mortality in men. Eur Heart J 36: 2706-2715. 
Citation: Sharma R, Oni O, Wiegmann P, et al. (2020) Testosterone Replacement Therapy (TRT) is Associated with Delayed Progression of Chronic Kidney Disease: A Retrospective Analysis of Testosterone Normalization in US Veterans. Ann Nephrol 5(1):51-59

14. Coresh J, Astor BC, Greene T, et al. (2003) Prevalence of chronic kidney disease and decreased kidney function in the adult US population: Third national health and nutrition examination survey. Am J Kidney Dis 41: 1-12.

15. Robins JM, Hernan MA, Brumback B (2000) Marginal structural models and causal inference in epidemiology. Epidemiology 11: 550-560.

16. Austin PC (2011) An introduction to propensity score methods for reducing the effects of confounding in observational studies. Multivariate behavioral research 46: 399-424.

17. Kittikulsuth W, Sullivan JC, Pollock DM (2013) ET-1 actions in the kidney: Evidence for sex differences. Br J Pharmacol 168: 318326.

18. Niemczyk S, Niemczyk L, Romejko-Ciepielewska K (2012) Basic endocrinological disorders in chronic renal failure. Endokrynol Pol 63: 250-257.

19. Gozukara KH, Arslan A, Gorur S, et al. (2015) Glomerular hyperfiltration in hypogonadotropic hypogonadic patients: Overlooking a cache? Int Urol Nephrol 47: 1099-1103.

20. Liu PY, Swerdloff RS, Veldhuis JD (2014) Clinical review 171: The rationale, efficacy and safety of androgen therapy in older men: Future research and current practice recommendations. J Clin Endocrinol Metab 89: 4789-4796.

21. Vecchio M, Navaneethan SD, Johnson DW, et al. (2010) Treatment options for sexual dysfunction in patients with chronic kidney disease: A systematic review of randomized controlled trials. Clin J Am Soc Nephrol 5: 985-995.

22. Vigen R, O'Donnell Cl, Baron AE, et al. (2013) Association of testosterone therapy with mortality, myocardial infarction, and stroke in men with low testosterone levels. J Am Med Asso 310: 1829-1836.

23. Finkle WD, Greenland S, Ridgeway GK, et al. (2014) Increased risk of non-fatal myocardial infarction following testosterone therapy prescription in men. PLoS One 9: e85805.

24. Basaria S, Coviello AD, Travison TG, et al. (2010) Adverse events associated with testosterone administration. N Engl J Med 363: 109-122.

25. Khurana KK, Navaneethan SD, Arrigain S, et al. (2014) Serum testosterone levels and mortality in men with CKD stages 3-4. Am J Kidney Dis 64: 367-374.

26. Morgentaler A, Feibus A, Baum N (2015) Testosterone and cardiovascular disease--the controversy and the facts. Postgrad Med 127: 159-165.

27. Morgentaler A, Miner MM, Caliber M, et al. (2015) Testosterone therapy and cardiovascular risk: Advances and controversies. Mayo Clin Proc 90: 224-251.

28. Budoff MJ, Ellenberg SS, Lewis CE, et al. (2017) Testosterone treatment and coronary artery plaque volume in older men with low testosterone. JAMA 317: 708-716.

29. Sharma R, Oni OA, Gupta K, et al. (2017) Normalization of testosterone levels after testosterone replacement therapy is associated with decreased incidence of atrial fibrillation. J Am Heart Assoc 6.
30. Sharma R, Oni OA, Chen G, et al. (2016) Association between testosterone replacement therapy and the incidence of DVT and pulmonary embolism: A retrospective cohort study of the veterans administration database. Chest 150: 563-571.

31. Shores MM, Smith NL, Forsberg CW, et al. (2012) Testosterone treatment and mortality in men with low testosterone levels. J Clin Endocrinol Metab 97: 2050-2058.

32. Baillargeon J, Urban RJ, Kuo YF, et al. (2014) Risk of myocardial infarction in older men receiving testosterone therapy. Ann Pharmacother 48: 1138-1144.

33. Muraleedharan V, Marsh H, Kapoor D, et al. (2013) Testosterone deficiency is associated with increased risk of mortality and testosterone replacement improves survival in men with type 2 diabetes. Eur J Endocrinol 169: 725-733.

34. Zhao JV, Schooling CM (2016) Endogenous androgen exposures and ischemic heart disease, a separate sample Mendelian randomization study. Int J Cardiol 222: 940-945.

35. Calof OM, Singh AB, Lee ML, et al. (2005) Adverse events associated with testosterone replacement in middle-aged and older men: A meta-analysis of randomized, placebo-controlled trials. J Gerontol A Biol Sci Med Sci 60: 1451-1457.

36. Jones SD, Dukovac T, Sangkum P, et al. (2015) Erythrocytosis and polycythemia secondary to testosterone replacement therapy in the aging male. Sex Med Rev 3: 101-112.

37. Bachman E, Feng R, Travison T, et al. (2010) Testosterone suppresses hepcidin in men: A potential mechanism for testosterone-induced erythrocytosis. J Clin Endocrinol Metab 95: 47434747.

38. Carrero JJ, Barany P, Yilmaz MI, et al. (2012) Testosterone deficiency is a cause of anaemia and reduced responsiveness to erythropoiesis-stimulating agents in men with chronic kidney disease. Nephrol Dial Transplant 27: 709-715.

39. DeGowin RL, Lavender AR, Forland M, et al. (1970) Erythropoiesis and erythropoietin in patients with chronic renal failure treated with hemodialysis and testosterone. Ann Intern Med 72: 913-918.

40. Kapoor D, Goodwin E, Channer KS, et al. (2006) Testosterone replacement therapy improves insulin resistance, glycaemic control, visceral adiposity and hypercholesterolaemia in hypogonadal men with type 2 diabetes. Eur J Endocrinol 154: 899-906.

41. Hackett G, Cole N, Bhartia M, et al. (2014) Testosterone replacement therapy improves metabolic parameters in hypogonadal men with type 2 diabetes but not in men with coexisting depression: The BLAST study. J Sex Med 11: 840-856.

42. Jones TH, Arver S, Behre HM, et al. (2011) Testosterone replacement in hypogonadal men with type 2 diabetes and/or metabolic syndrome (the TIMES2 study). Diabetes Care 34: 828-837.

43. Kapoor D, Aldred H, Clark S, et al. (2007) Clinical and biochemical assessment of hypogonadism in men with type 2 diabetes: Correlations with bioavailable testosterone and visceral adiposity. Diabetes Care 30: 911-917.

44. Snyder PJ, Bhasin S, Cunningham GR, et al. (2016) Effects of testosterone treatment in older men. N Engl J Med 374: 611-624.

DOI: $10.36959 / 832 / 400$

Copyright: (C) 2020 Sharma R. This is an open-access article distributed under the terms of the Creative Commons Attribution License, which permits unrestricted use, distribution, and reproduction in any medium, provided the original author and source are credited. 